\title{
Das Eigene und das Fremde in der Logik von Hierarchisierung und Dominanz in Gottfrieds Tristan
}

\author{
Monika Schulz
}

\begin{abstract}
The study exemplifies with Gottfried's Tristan, how subtle medieval texts handle intercultural coded, but distinctly different social structures (here: gender) by strategies of inclusion and exclusion, respectively, by spatial arrangement (>Heterotopia<). The >Own< (i.e., the essential patriarchal structure of courtly culture) and the >Foreign< (i.e., the gendersymmetric social structure in Gottfried's Ireland) are arranged into a system of hierarchy and dominance. Gottfried's >Foreign < is not a simple fairytale-like alterity of his >Own<; rather, within the >Foreign < he deals with the prominent gender-discourse of early Irish legal documents (Cáin lánama) and myth-analogous narratives (e.g. Táin Bó Cuailnge). While Gottfried's >Foreign< can be connected to the courtly code (i.e., the >Own<) by the ideal of the courtly vrouwe, respectively, while the $>$ Foreign < does not reject the courtly infiltration, this compatibility cannot be detected at all in Gottfried's precursor Eilhart; his narrated world is a laic warrior society.
\end{abstract}

Title: The >Own and the >Foreign $<$ in the Logic of Hierarchy and Dominance in Gottfried's Tristan

Keywords: Gottfried von Straßburg (-ca. 1215); Tristan; Isolde; Táin Bó Cuailnge; gender

\section{Voraussetzungen}

Im Folgenden soll das komplexe interkulturelle Setting in Gottfrieds Tristan beleuchtet werden. Es geht um das Verhältnis von Eigenem und Fremdem bzw. Anderem, ${ }^{1}$ um Strategien der Inklusion und Exklusion (vgl. Gutjahr 2010: 33). Textlogisch meint das >Eigene < hier die höfische Kultur ${ }^{2}$ als Verständigungsraum der Elite des europäischen Mittelalters mit ihrer basal patriarchalen Struktur (vertreten durch König Marke in Cornwall), das >Fremde < ist verortet im nichtpatriarchalen, geschlechtssymmetrisch angelegten Irland mit der superioren

1 Bekanntlich ist "der zentrale Gegenstand der Interkulturalitätsforschung die Frage nach dem Fremden " (Heimböckel/ Mein 2010: 9).

2। Mein Kulturbegriff ist in der Nachfolge von Geertz ein bewusst weit und allgemein gefasster und ein wesentlich semiotischer; Geertz formuliert wie folgt: "Ich meine mit Max Weber, daß der Mensch ein Wesen ist, das in selbstgesponnene Bedeutungsgewebe verstrickt ist, wobei ich Kultur als dieses Gewebe ansehe“ (Geertz 1987: 9). 
Aktantin Königin Isolde an der Spitze. Nur Gottfried hat diese interkulturell codierte Dramaturgie konkurrierender Geschlechterentwürfe; Eilharts vorausgehende Version kennt keine solche Doppelgleisigkeit, denn Markes Reich sowie Irland sind hier gleichermaßen patriarchal, das >Fremde $<$ im vorgestellten Sinn ist vollständig ausgeblendet.

Wenn man mit Lotman Gottfrieds Text als semiotischen Raum im Sinn einer »Semiosphäre « (Lotman 1990: 287) liest, die ein >Innen < (hier sind die »dominierenden semiotischen Systeme angesiedelt«, ebd.: 294) und ein >Außen kennt, eine Opposition Zentrum/Peripherie (vgl. ebd.: 293; übersetzt ein >Eigenes $<$ und ein >Fremdes $<$ also), ist seine Beobachtung von >Grenze $<$ und $>$ Filtration< (vgl. ebd.: 292) von Interesse. Das >Innen< meint Gottfrieds höfischen Kulturraumentwurf (das Reich Markes), der zweifellos auch territorialen Charakter hat: Die prominente Grenze zum fremden Raum im >Außen < (Irland) bildet das Meer als intermediärer Schwellenraum, ${ }^{3}$ der als Puffer »eine räumliche Funktion im elementaren Sinne« (Lotman 1990: 292) hat. Die Grenzüberschreitung ist dem Helden vorbehalten ${ }^{4}$ - und selbst diese ist bei Gottfried noch kulturspezifisch markiert. Denn während Eilharts Tristan sich ganz im Sinne der keltischen immram ziellos auf dem Meer treibend Irland nähert (dies ist auch so bei Thomas bzw. in der Tristrams Saga), differenziert Gottfried auffällig: Zunächst verläuft Tristans Reise dank der Hilfe eines kundigen (höfischen) Steuermanns wohlgeordnet zielgerichtet, erst eine halbe Meile vor Dublin wird Tristan auf eigenen Wunsch auf dem wilden Meer in einem Boot treibend zurückgelassen (v. 7492-7494)5. Tristan ist so scheinbar zufällig Entdecker des Fremden bzw. Anderen, wobei Grenzüberschreitung und Fremdbegegnung doppelt codiert sind: Als Moribunder benötigt er die zauberische Heilkraft der Königin (ist also existentiell auf das Fremde angewiesen und >Nehmer< des Fremden) und ist gleichzeitig > Geber < des Eigenen, wenn er die junge Isolde in höfisch-ethischer Erziehung unterweist. Dieser scheinbar paritätische Kulturaustausch folgt in Wahrheit der Logik von Hierarchisierung und Dominanz: Bei der ersten Fremdberührung, beim Kampf mit Isoldes Bruder Morold, der wohl nicht zufällig auf einer zu Markes Land gehörigen Insel, also noch im höfischen Raum, stattfindet, wird Tristan zunächst durch das Fremde tödlich infiziert, er wird nicht nur metaphorisch, sondern ganz buchstäblich vergiftet. Der Sieg Tristans über Morold dann meint den Sieg des Höfischen über das >Andere <, das sogleich aus dem eigenen Raum entfernt wird: Der segmentierte Körper Morolds wird in die Fremde zurückgeschickt.

3 | Vgl. zu 'Schwellenraum` und 'Grenzüberschreitung، van Gennep 2005: vor allem 29.

4 | Das ist durchaus nicht ungewöhnlich; denn dass "der Mann in die Fremde muss, um die Mission seiner Selbstfindung zu erfüllen, ist als wirkungsmächtiges geschlechtsspezifisches Muster der abendländischen Literatur vom griechischen Epos über den höfischen Roman bis zum Bildungsroman eingeschrieben " (Gutjahr 2010: 33).

5 | Zitiert wird in diesem Beitrag nach Gottfried von Straßburg 2011. 
Es ist signifikant, dass die zauberische Heilhandlung der irischen Königin (wie auch ihr Minnetrank und die prophetischen Wahrträume) nicht in der höfischen Sphäre (Cornwall) situiert sind, wie überhaupt die irische Königin als handlungsmächtige Aktantin der fremden Welt diese nie verlässt und nur dort agiert; ein Transfer der Spitzenvertreterin des >Anderen< in die höfische Welt (Cornwall) scheint unmöglich. Ein solcher Transfer gelingt erst in der nächsten Generation; Voraussetzung hierfür ist eine weitreichende Infiltration des textlogisch >Fremden $<$ durch das >Eigene $<-$ und so ist es auch kein Zufall, dass der Deal für den Austausch von Irland ausgeht (die Königin macht bekanntlich die Heilung Tristans von der höfischen Unterweisung ihrer Tochter abhängig): Das textlogisch >Fremde<, hier vertreten durch Königin Isolde, fordert also von sich aus das für sie Fremde, aber textlogisch >Eigene< ein und anerkennt damit dessen Superiorität (im Gegensatz zu Morold, der das mit dem Leben bezahlt). Wohl unterrichtet in der morâliteit durch den höfischen Kulturvermittler Tristan, kann die irische Prinzessin dann in den für sie fremden Kulturraum (Cornwall) überstellt und damit in das textlogisch >Eigene< inkorporiert werden. Die Differenzzuschreibung scheint gelöscht, in Wahrheit aber sind die kulturdistinktiven Indizes der transferierten Prinzessin so geschickt transformiert, dass sie problemlos an den höfischen Code, an das dominierende semiotische System also, anschließbar sind. ${ }^{6}$

In gewisser Hinsicht ist Gottfrieds Irland im Sinne Foucaults ein »Or[t] außerhalb aller Orte« (Foucault 1992: 39), wiewohl er tatsächlich geortet werden kann; er ist eine Heterotopie, genauer: eine >Abweichungsheterotopie ${ }^{7}$, ein privilegierter Sonderraum (noch dazu eine Insel), der eine soziale Struktur erlaubt, die im textlogisch >eigenen< Territorium, im höfischen Cornwall Markes, nicht denkbar wäre. Genau besehen ist jedoch bereits Cornwall ein Grenzort (Halbinsel), der sozusagen als eine Art >Vermittlungsheterotopie< für die Kontaktaufnahme mit dem >Fremden $<$ prädestiniert ist.

6 | Denn genau besehen, hat auch in Markes Reich die Frau (die junge Isolde) die Fäden in der Hand; ihre Superiorität ist jedoch mit dem höfischen Code vereinbar, ist sie doch Meisterin im Listhandeln bzw. der doppeldeutigen Rede (Baumgartenszene, Gottesurteil etc.). Vor der Folie des zeitgenössischen prudentia-Diskurses wurde (in der hochhöfischen Epik) "das Ideal der 'schoenen، List durchaus erlaubt, als Bestandteil einer spezifisch höfischen Form der Konfliktlösung, welche Diplomatie als hohen Wert ansieht" (Semmler 1991: 233).

7 | 'Abweichungsheterotopier meine ich hier nicht streng im Sinne Foucaults, der darunter Orte wie etwa Gefängnisse versteht und wie folgt definiert: "In sie [die Abweichungsheterotopien; M.S.] steckt man die Individuen, deren Verhalten abweichend ist im Verhältnis zur Norm " (Foucault 1992: 40). Ich dagegen lege in der Verwendung des Begriffs den Fokus auf die 'Abweichung، selbst, die ich mir im Unterschied zu Foucault als nicht zwangsläufig im reigenen، Raum angesiedelt vorstelle. 


\section{INKLUSIONSSTRATEGIEN}

Gottfrieds >Fremdes $<$ ist nicht lediglich eine märchenhafte Alteritätsrelation zum $>$ Eigenen $<$, er verhandelt hierin geschickt prominente Geschlechterdiskurse des frühen Irland (dem Raum also, in dem Gottfrieds >Fremdes< angesiedelt ist). Trotz der Inklusion des fremden Registers (freilich räumlich ausgelagert, Stichwort: Heterotopie) lassen Überblendungen auch das Figurenpersonal im fremden Raum durchaus noch als höfisch erscheinen (die irische Königin ist trotz ihrer Fremdheit immer auch eine höfische vrouwe). Bei Gottfried umfassen die Episoden, in denen diese eine Schlüsselrolle besitzt, etwa 4300 Verse, also mehr als ein Fünftel (vgl. Rasmussen 2003: 142) von seinem Torso. Nur Gottfried entwickelt die bifigurale Isoldekonzeption, Eilhart dagegen gestand seiner irischen Königin nur wenige Verse zu. Vor allem die genderorientierte Forschung betonte die »ungewöhnlichen Befugnisse « (Kellermann-Haaf 1986: 71) von Gottfrieds Königin, die intelligenten Operationen der irischen Frauen (die beiden Isolden und Brangäne) stünden im Kontrast zur dominierenden mittelalterlichen klerikalen Ideologie einer weiblichen Passivität und Schwäche, ${ }^{8}$ Gottfrieds Irland sei »women's space« (Sterling-Hellenbrand 2001: 168). Das ist zweifellos alles richtig, erklärt jedoch nicht, woraus sich Gottfrieds auffällige Irlandkonzeption speist. Will man sich Gottfrieds >Anderem « in nuce nähern, ist das nicht ohne Weiteres, d.h. hier nicht ohne dezidiert transdisziplinäre Anleihen, möglich. Erst in Kenntnis relevanter Rechtstexte und mythosanaloger Narrative des keltischen Irland - die ob ihrer Prominenz und Brisanz auch auf dem Kontinent mindestens als ungefähres Wissen innerhalb der zeitgenössischen (literaten) Elite zirkuliert sein müssen - wird Gottfrieds Umgang mit dem >Fremden Verfahren der Infiltration und Depotenzierung durch das >Eigene< erfahrbar. Es wird also im Folgenden zunächst um die Decodierung dieses >Fremden < gehen müssen: um die Ermittlung von Kulturcodes als >Hypotext< und ihrer Transposition bei Gottfried. Eine Annäherung kann nur vermittels eines close reading im Geertz'schen Sinne (vgl. Geertz 1973: 453) erfolgen.

\subsection{Frauenbilder der Keltologie}

Die einschlägige keltologische Forschungsliteratur zur Stellung der Frau im frühen Irland ist zunächst irritierend. Die ältere Forschung geht von einer Art Matriarchat in der Frühzeit aus, wobei bei den Inselkelten eine Wende zum Patriarchat im ersten nachchristlichen Jahrhundert angenommen wird; dies sei gut in den literarischen Zyklen ablesbar, in denen vor der Wende König Conchobar eben als >Sohn der Mutter < (mac Nessa) bezeichnet werde, dagegen

8 So Rasmussen: "The agency and intelligent resolve shown by all the female figures - Blanscheflur, Tristan's mother; princess Isolde and her mother, Queen Isolde; brangaene, Princess Isolde's cousin and companion - stand in contrast to the dominant medieval clerical ideology of femine passivity and weakness" (Rasmussen 2003: 137). 
ChuChulinn >Sohn des Vaters< (mac Sualtam) sei (vgl. Clarus 1991: 118-120); auch der Thron sei in der Frühzeit über die weibliche Linie weitergegeben worden (vgl. ebd.: 120). Die jüngere Forschung distanziert sich teilweise vehement von Einschätzungen, die in Richtung >Matriarchat< etc. gehen (dabei ist längst erkannt, dass Bachofen und Morgan denselben Fehler machten, indem sie von der Matrilinearität auf ein Matriarchat schlossen, vgl. zu diesem Sachverhalt Wesel 1980: vor allem 19-25). Patterson stellte in seinem Bemühen, die frühe irische Gesellschaft zu rekonstruieren, klar heraus, dass die irische Überlieferung selbst Zeugnis ablegt von der Teilnahme von Frauen in Politik und Kriegsführung, sofern sie zur Elite gehörten (vgl. Patterson 1991: 25f.). Den Aufwand, der innerhalb der Celtic Studies betrieben wurde, um zu zeigen, dass die irischen Frauen >ursprünglich< keine rechtliche Autonomie hatten, kommentierte Patterson mit der Bemerkung, dass die Celtic Studies ihrerseits nicht frei von patriarchalischen Einstellungen seien (vgl. ebd.: 26). Dazu gehört auch die Tendenz einiger Keltologen, generell nicht von weiblichen Anführerinnen, sondern von Göttinnen auszugehen, etwa wenn der Sachverhalt bewertet wird, dass Eponyme zahlreicher vorchristlicher sozialer Gruppen weiblich waren (vgl. ebd.).

Selbstverständlich ist zwischen den Frauen in den Sagas, also in der Literatur, und den Frauen der außerliterarischen Realität zu unterscheiden, wobei Letztere unzweifelhaft eingeschränkter waren (vgl. dazu Kelly 1991: 68f.), ${ }^{9}$ vor allem dann, wenn sie nicht der Elite angehörten. Greens Hinweis ist bedenkenswert: »[E]ven if there is evidence for a few powerful female leaders within a particular culture, that need not correlate with the position of women in general« (Aldhouse-Green 1995: 15). Doch zweifellos gab es unabhängige Frauen im frühen Irland, die der Elite angehörten und dies entweder über Besitz oder über einen besonderen Status (Dichterinnen, Ärztinnen) erreichten (vgl. Kelly 1991: 77). Erschwert scheint die Einschätzung auch dadurch, dass es in den kirchenrechtlichen Sammlungen zwei Hauptrichtungen gibt, was die Stellung der Frau in der irischen Gesellschaft betrifft: Für die Romani, das waren die Kleriker im Süden Irlands, die die irische an die paternalistische Gesellschaft der Bibel anpassen wollten, war die Frau immer unter männlicher Vormundschaft (Vater, Ehegatte, Sohn); bei den Hibernenses, das waren die Kleriker im Norden Irlands, die die christliche Religion in die bestehende Gesellschaft integrieren wollten, hatten die Frauen große Rechte. ${ }^{10}$ Beide, Romani und Hibernensens, waren übrigens »nicht bereit, das in Irland praktizierte weltliche Eherecht zu tolerieren« (Richter 1996: 95). Für sich spricht die Existenz der Gattung des Ban-Shenchus, d.h. so viel wie >Frauen-Geschichte < bzw. Frauen-Überlieferung. Das sind lange Listen berühmter Frauen, die in den Handschriften zusammengestellt wur-

9 | Nicht ohne Polemik hat das Bitel folgendermaßen formuliert: "These stay-at-home legal incometents were the real Medbs [das meint die allmächtige Königin im ,Rinderraub von Cooley،; M.S.] of early Ireland " (Bitel 1996: 10).

10 | Für diese Information auf eine entsprechende Nachfrage hin danke ich Prof. Dr. Doris Edel. 
den, zum Teil in Versform und mit kurzen Charakteristiken der Frauen (vgl. Weisweiler 1939: 237).

\subsection{Die ’Regelung der Paare، (Cáin lánama) im frühen irischen Eherecht}

Es empfiehlt sich der unmittelbare Rekurs auf altirische Rechtsdenkmäler. ${ }^{11}$ Diese bieten zwar nur »das Bild einer Ruine, um nicht zu sagen eines Trümmerhaufens « (Thurneysen 1935: 267), auch fehlen ergänzende Urkunden aus der älteren Zeit (vgl. ebd.), doch sei eben der »Reiz des alten Irland [...], daß wir dort ein Rechtsleben antreffen, das wir anderwärts meist nur als Grundlage, als Vorstufe erschließen können, das dort aber in gleichzeitigen Rechtstexten klar bezeugt ist « (ebd.: 286). Zu bedenken ist, dass das irische Recht »kein völlig unbeeinflusstes keltisches Recht [ist]. Alle Texte stammen aus christlicher Zeit, und den Einfluß der Kirche, die im hl. Patricius verkörpert wird, betonen einige selber« (ebd.: 281); die erhaltenen Handschriften sind jung (die meisten stammen aus dem 14. bis 16. Jahrhundert), es wird jedoch davon ausgegangen, dass die Kompilation der Rechtssammlung schon im 8. Jahrhundert vorhanden war, wie Auszüge aus Glossierungen zeigen; die einzelnen Texte scheinen womöglich dem 7 . Jahrhundert anzugehören (vgl. ebd.: 270).

Für unseren Zusammenhang ist die sogenannte >Regelung der Paare (Cáin lánama) relevant; vollständig erhalten ist der Text mit Glossen und Kommentar in einer Handschrift aus dem 14. Jahrhundert (vgl. ebd.: 287). Die >Regelung formuliert deutlich das Kriterium für eine Vormachtstellung der Frau: Diese ist abhängig von ihrem in die Ehe eingebrachten Besitz. Es gibt vereinfacht vier $>$ Paarungen $<$ :

1. Paarung mit gemeinsamem Einbringen: »[W]enn sie (die Paarung, die Ehe) mit Land und Vieh und Wohnungsausstattung (Geräten) ist, und wenn ihre (der Gepaarten) Paarungs-Gleichheit von gleichem Adel, von gleicher Gebührlichkeit ist (d.h. wenn ihre Gleichheit für die und in der Ehe auf gleichem Stande und gleichem gebührendem Betragen beruht) - und eine solche Frau wird >Frau der Mitherrschaft< genannt -, ist kein Vertrag von einem der beiden Teile ohne den anderen ein (gültiger) Vertrag « (Thurneysen 1936: 305).

2. Paarung einer Frau auf Manneseinbringen: »[E]in Vertrag des Mannes ohne die Frau ist ein (gültiger) Vertrag [...], wenn sie (zwar) eine angetraute Frau, aber nicht Hausvorstand ist« (ebd.: 332). Hausvorstand meint 〉Vorstand des Haushalts< (cémuinter), eine solche Frau kann »zwar nicht selbständig Ver-

11 | Für die ausgesprochen wertvollen und unverzichtbaren Hinweise auf relevante keltologische Primär- wie Sekundärliteratur danke ich sehr herzlich Prof. Dr. Stefan Zimmer; ohne seine Hilfe wäre ein ,Wildernı im interdisziplinären fremden Raum nicht möglich gewesen. 
träge schliessen wie die >Frau der Mitherrschaft<, sondern nur ihr Mann, wohl aber hat sie Einspruchsrecht gegen alle seine Verträge« (ebd.: 333).

3. Paarung eines Mannes auf Fraueneinbringen: »[B]ei dieser geht der Mann auf der Bahn der Frau [...] und die Frau auf der Bahn des Mannes« (ebd.: 343). Die Pflichten eines solches Ehemannes ohne Einbringen sind dahingehend, dass er ein >Mann des Dienstes $<$ sein soll und ein >Haupt des Rates< (vgl. ebd.). ${ }^{12}$

4. Paarung eines $>$ Mannes des Besuchens $<$ : Während es sich bei den ersten drei Beziehungen um so etwas wie Ehen handelt, betrifft die vierte Form »weniger reguläre Verhältnisse« (ebd.: 350), die hier nicht weiter interessieren.

Fazit: Die >Paarung eines Mannes auf Fraueneinbringen< ist nach frühem irischen Recht Voraussetzung für die Herrschaft der Frau innerhalb der Ehe, hier geht der Mann »auf der Bahn der Frau« (ebd.: 343).

\subsection{Isolde und Medb: Königliche Ehefrauen eines Mannes sauf der Bahn der Frauı}

Gottfrieds irische Königin wurde in der Forschung als »political leader and diplomat« (Rasmussen 2003: 139) Irlands bezeichnet; sie hält in der entscheidenden Gerichtsszene (Truchsess) alle Fäden in der Hand, rettet damit die Ehre des Königshauses und eben nicht ihren Ehemann, König Gurmun. Das ist keine bloß phantastische Setzung, Gurmun ist in der Terminologie des frühen irischen Rechts eben nur ein Mann, der >auf der Bahn der Frau< geht. Das ist bei Gottfried so vermittelt, dass Isoldes Ehemann ein ursprünglich landfremder Eindringling ist, der auf die Allianz mit einer mächtigen irischen Familie angewiesen ist (siehe weiter unten). Ähnliche Konstellationen werden in mythopoetischen Erzählungen der frühen irischen Literatur ausführlich verhandelt (es geht dabei selbstverständlich nicht um direkte Abhängigkeiten): Die über ein Jahrtausend tradierte Táin Bó Cúailnge, der >Rinderraub von Cooley<, der längste und bedeutendste Text des Ulsterzyklus, ${ }^{13}$ einer der vier großen Zyklen der mittelalterlichen irischen Literatur, erzählt von der Herrschaft einer Frau eines >Mannes auf Fraueneinbringen $<$. Das Gerangel um die Vormachtstellung ist da-

12 | Unter Ersterem verstehen Glosse und Kommentar "das Betreiben, Anordnen des Pflügens (aradu air) und die Hälfte der Besorgung der Wirtschaft (frichnam na trebaire). Ein ,Haupt des Rates، ist er, wenn er bei den Beratungen eine führende Stellung hat und seinem Rate zu folgen ist" (Thurneysen 1936: 344).

13 | Der Ulsterzyklus heißt so, weil die Mehrzahl seiner Figuren zum alten Königreich von Ulster gehören; das vorchristliche Irland, das den eigentlichen Hintergrund des Zyklus formt - so wird z.B. noch von der Kopfjagd erzählt, die Sammlung von Köpfen spiegelt die Bedeutung des Helden (vgl. dazu Richter 1996: 26) -, bestand aus fünf unabhängigen Teilen: Ulster, Connacht, Leinster, Munster und Mide (vgl. dazu Edel 2001: 153). 
bei das Thema, wobei das entscheidende Kriterium der Besitz ist (ganz analog also zu den Vorgaben in der >Regelung der Paare $<)$.

Der >Rinderraub von Cooley< umfasst je nach Fassung 4000 bis 5000 Zeilen. Möglicherweise bietet das Epos den »besten Zugang zu der Oberschicht Irlands in christlicher Zeit« (Richter 1996: 24). In seinem Kern geht das Material des Rinderraubs wohl auf die Zeit um 700 n.Chr. zurück (vgl. Edel 2001: 161), es »wird allgemein angenommen, daß heute verschollene ältere schriftliche Fassungen bis ins 7. Jh. zurückreichten« (Richter 1996: 24). Die Textentwicklung »lässt sich von der altirischen Periode bis ins 15. Jahrhundert verfolgen, ihre handschriftliche Überlieferung vom 11. bis zum 19. Jahrhundert« (Edel 2012: 139). Die älteste Version ist die sogenannte Recension I (TBC I) (vgl. Edel 2001: 39). Jackson (vgl. 1964) hielt es für möglich, dass das Geschehen im >Rinderraub< auf das 4 . Jahrhundert n.Chr. datiert werden kann. Der >Rinderraub< schildert den Kampf zwischen den Provinzen Ulster und Connacht.

Ein historischer Hintergrund wird angenommen, vorsichtig formuliert das Edel: »The antagonism between Ulster and Connacht, which dominates the whole Ulster cycle, may have a base in historical fact « (Edel 2001: 39); Richter postuliert: »Der historische Rahmen ist durch die Abdrängung der Ulaid in die nordöstlichen Gebiete Irlands durch die Könige von Connacht gegeben, ein politischer Umwälzungsprozeß, der sich über mehrere Jahrhunderte erstreckte« (Richter 1996: 25). ${ }^{14}$

Zum Inhalt: Während Ulster von König Conchobor regiert wird, steht an der Spitze von Connacht (das ist eine der historischen Provinzen Irlands) nicht König Ailill, sondern seine Frau, Königin Medb. Sie entscheidet über Krieg und Frieden, sie hat den Oberbefehl über das Heer und kämpft auch selbst mit, wobei sie mehrere Gegner verwundet; dennoch gilt sie als sehr attraktive Frau. ${ }^{15}$ Medb reklamiert für sich die Position, die in einer patriarchalen Gesellschaft dem Ehemann zusteht (vgl. Edel 2001: 170), so zahlt sie auch den Brautpreis selbst ${ }^{16}$ und nicht ihr zukünftiger Ehemann, der eben nur ein >Mann auf Fraueneinbringen < ist. Das aber pervertiert die Geschlechterrollen, üblicherweise gibt

14 | Zum Aspekt der Archaisierung bzw. politischen Intentionen siehe Ó hUiginn 1993.

15 | Ihre Erscheinung wird folgendermaßen beschrieben: "She had long fair hair, and two golden birds on her shoulder. [...] She carried a light, stinging, sharp-edged lance, and she had an iron sword with a woman's grip" (Edel 2001: 162).

16 | It was I plighted thee, and gave purchase-price to thee, which of right belongs to the bride - of clothing, namely, the raiment of twelve men, a chariot worth thrice seven bondmaids, the breadth of thy face of red gold, the weight of thy left forearm of silvered bronze. Whoso brings shame and sorrow and madness upon thee, no claim for compensation nor satisfaction hast thou therefor that I myself have not, but it is to me the compensation belongs, said Medb, for a man dependent upon a woman's maintenance is what thou art. ([Táin Bó Cuailnge] 1914: 3) 
der Ehemann den Brautpreis an den Vater der Braut (vgl. Kelly 1991: 118; in seiner ursprünglichen Form wird er zwischen lineages geleistet und damit wird »lineage-Eigentum, kollektives Eigentum, übertragen«, Wesel 1980: 134; diejenige lineage, die ein weibliches Mitglied verliert, erhält einen Ausgleich). Für unseren Zusammenhang von Interesse ist das sogenannte >Kopfkissengesprächく. In der Rezension II (die Rezension I setzt die Motivation für den Rinderraub voraus) steht der > pillow-talk < zu Beginn, in dem Medb und ihr Eheman Ailill die Vorrangstellung jeweils für sich beanspruchen. Weil sie die Erbin von Connacht ist, nennt Medb ihren Ehemann Ailill fer ar bantinchur, einen Mann auf Fraueneinbringen.

Der >pillow-talk $<:^{17}$ Königin Medb und ihr Mann Ailill befinden sich im königlichen Gemach in der Festung von Connacht, als Ailill behauptet, dass seine Frau besser dran sei als vor ihrer Heirat. Die Königin antwortet, dass sie auch ohne ihn sehr gut dagestanden sei. Ailill antwortet, dass Medbs Reichtum so beschaffen gewesen sei, dass sie nichts darüber wussten oder hörten, außer, dass sie eine Erbtochter gewesen sei und dass ihre benachbarten Feinde sie ausgeraubt und geplündert hätten. Medb ist mit dieser Interpretation nicht einverstanden und gibt zur Antwort: Ihr Vater sei ardri von Irland gewesen, also Hochkönig, ${ }^{18}$ und sie sei die vornehmste und kriegerischste seiner sechs Töchter gewesen, mit einer mächtigen Streitmacht unter ihrem Kommando; ihr Vater habe ihr Connacht gegeben und deshalb sei ihr Ehemann Ailill fer ar bantinchur. Ailil antwortet, dass er ebenfalls von einer mächtigen Familie abstamme, er sei nach Connacht gekommen, um zu regieren, weil er nie von einer Frauenherrschaft (ar bantinchur) gehört habe außer von ihr und weil er selbst das Königtum von Connacht über seine Mutter beanspruche.

Um ihre Ansprüche zu rechtfertigen, vergleichen die Ehepartner die halbe Nacht Stück für Stück ihre jeweiligen Besitztümer, so etwa Kleidung und Schmuck, Pferde, Schweine und die Viehherden. Für jede Kategorie ist der Besitz in Anzahl und Größe gleich - jedoch mit einer Ausnahme: Unter Ailils Viehherde befindet sich ein außergewöhnlicher Bulle, der weißgehörnte Finnbenach, für den Medb kein Gegenstück besitzt. Dieser Bulle gehörte als Kalb einst ihr, er ging aber dann in Ailills Herde, weil er sich weigerte ar bantinchur zu sein, unter der Herrschaft einer Frau: Das Ausgangsthema wird hier also unübersehbar wiederholt. Medb hört von einem berühmten Bullen in Ulster, Donn Cúailnge, dem Braunen Bullen von Cooley. Der Versuch, den Bullen auszuleihen, misslingt, so dass Medb beschließt, den Bullen mit mililtärischer Gewalt an sich zu bringen (vgl. Edel 2001: 169; [Táin Bó Cuailnge] 1914: 6, 9).

17 | Vgl. [Táin Bó Cuailnge] 1914: 1-4.

18 | Vgl. zu ardrí Edel 2001: 171; vgl. zu Hochkönig Thurneysen 1935: 284. 
Der Kriegsgrund referiert Bedingungen des frühen Irland: Reichtum und soziale Stellung wurden über die Zahl der Rinder hergestellt; selbst der sogenannte >Ehrenpreis< (enech, urspr. >Gesicht<, vergleichbar dem germ. Wergeld) wurde in den Rechtstexten in Rindern angegeben (vgl. Richter $1996: 33) .{ }^{19} \gg$ In the early period at least, the aim of a military expedition was generally not to annex the opponent's land, but to demonstrate one's own superiority by carrying of his cattle« (Edel 2001: 158). Zudem ist der Braune Bulle wie sein Gegenstück Finnbennach »das Resultat mehrerer Metamorphosen eines magischen Schweinehirten, [...] unter Beibehaltung seiner menschlichen Intelligenz ist er zu einem gewaltigen Tier geworden - fünfzig Halbwüchsige können seinen Rücken gleichzeitig als Spielfeld benützen« (Botheroyd/Botheroyd 1992: 88).

Auf Befehl Königin Medbs werden die Truppen von Connacht mobilisiert und sie beginnt einen Angriffskrieg gegen Ulster mit der Unterstützung verbündeter Länder. Die Kämpfe interessieren in unserem Zusammenhang nicht weiter; die Invasion Medbs und ihrer Verbündeten schließt damit, dass Medb dem berühmten Helden von Ulster, Cu Chulainn, unterliegt; Medb bittet um Schonung und $\mathrm{Cu}$ Chulainn sieht davon ab, sie zu töten, weil er keine Frauen töte. Das wird von Fergus, Medbs Liebhaber, folgendermaßen kommentiert: And even as a boordmare leads her foals into a land unknown, without a head to advise or give counsel before them, such is the plight of this host today in the train of a woman that hath ill counselled them ([Táin Bó Cuailnge] 1914: 362). Die Forschung las diese Stelle dahingehend, dass die literati, auf die die erste Rezension des Rinderraubs zurückgeht, >robuste Antifeministen< gewesen seien (vgl. Bowen 1974: 30).

Am Schluss steht der Kampf der beiden Bullen, den z.B. Greene als eines der zentralen Motive des Epos sieht (vgl. Greene 1959: 99). Alle Überlebenden verfolgen begierig den Verlauf; der Braune Bulle von Ulster geht als Sieger hervor; im Morgengrauen zieht er fort, mit den Überresten Finnbennachs auf den Hörnern, die er an verschiedenen Orten fallen lässt; daraus entstehen dann Ortsnamen wie >Furt der Leber < oder ,Bach des Schulterblattes«; schließlich aber stirbt er vor Erschöpfung. Ailill und Medb schließen Frieden mit den Männern von Ulster und Cu Chulainn (vgl. [Táin Bó Cuailnge] 1914: 369).

Königin Medb fand großes Interesse innerhalb der keltologischen Forschung. Nach Ross verkörpert sie als Herrscherin, Mutter, Politikerin, Strategin und

19 | Medbs Ehebedingungen, die sie auch Ailill gestellt hat (der Mann darf nicht geizig oder eifersüchtig und muss furchtlos sein) begründet sie folgendermaßen: Were my husband a coward, 'twere as unfit for us to be mated, for I by myself and alone break battles and fights and combats, and 'twould be a reproach for my husband should his wife be more full of life than himself, and no reproach our being equally bold. ([Táin Bó Cuailnge] 1914: 3) 
Schlachtenführerin den Typus einer keltischen Königin, die als irdisches Spiegelbild einer göttlichen Magna Mater gesehen werden könne (vgl. Ross 1967: 360). Egeler interpretierte im Gefolge Thurneysens Medb als euphemerisierte Göttin und sah im Vergleich mit altorientalischem, klassisch-mediterranem und nordischem Material unter den Parametern >Krieg, Sexualität bzw. erotische Aktivität, Königtum und Vogelattribut< Parallelen mit Inanna-Ištar, Aphrodite, Venus und Freyja (vgl. Egeler 2012: 77-86). Ende des letzten Jahrhunderts übrigens bescheinigten Keltologen Medb Dominanz, Unmoral und sexuelle Unersättlichkeit, was dem Ruf der irischen Frau in der öffentlichen Meinung Englands schade (vgl. Botheroyd/Botheroyd 1992: 222). ${ }^{20}$

Zurück: Isoldes Machtfülle bei Gottfried gründet darin, dass sie eine reiche Erbin und der ursprünglich landfremde Ehemann nur ein »Mann auf der Bahn der Frau « ist. Ein Indiz hierfür ist bereits der matrilokale Wohnsitz. Eine solche Residenzregelung ist oft Merkmal matrilinearer Gesellschaften, in der die Kinder zur lineage der Frau gehören (vgl. Wesel 1980: 135). Mälzer sah überhaupt in der besonderen Schwester-Bruder-Beziehung (Königin Isolde und Morold bzw. die Prinzessin und ihr Mutterbruder Morold) einen Hinweis für eine sarchaisch-matriarchale< Ausrichtung des irischen Königshofs (vgl. Mälzer 1991: 83). ${ }^{21}$ Dass nun aber eine solche avunkulare Struktur das gesamte Geschehen steuert (bekanntlich will König Marke als >Mutterbruder< das Kind seiner Schwester, Tristan also, zum Erben einsetzen), wirft die Frage auf, ob nicht das textlogisch $>$ Fremde $<$ Gottfrieds das ursprünglich $>$ Eigene $<$ gewesen ist.

Die irische Isolde tritt bei Gottfried zum ersten Mal in Erscheinung in $\mathrm{Zu}$ sammenhang mit Ereignissen der Landnahme, wobei reale Bedingungen zum Teil genau referiert sind; Gottfried war offenbar mit Irlands (kultur-)historischen

20 | Die Frage übrigens, ob es reale 'Kriegsfrauenı gab, beantwortet die Forschung oft mit dem Hinweis auf Beschreibungen der klassischen Autoren. So berichtet etwa der griechische Hisotriker Dio Cassius (150-235 n.Chr.) von boudicca, Königin eines keltischen Stamms, die ihre Leute in eine blutige Revolte gegen die Römer geführt haben soll; nach Ammianus Marcellinus (330-395 n.Chr.) sind die keltischen Frauen überhaupt kampflüstern und er beschreibt übertreibend die Frau eines Kriegers, weitaus stärker als er, mit blitzenden Augen, knirschenden Zähnen, Schläge und Stöße mit ihren riesigen weißen Armen austeilend wie Schüsse, die von einem Katapult abgefeuert werden (vgl. Edel 2001: 41). In einer irischen Synode, 679 n.Chr., wurde Frauen verboten, sich aktiv an militärischen Operationen zu beteiligen (vgl. ebd.).

21 | Dass das Sippendenken des frühen Irland die ungewöhnlichen Machtbefugnisse der irischen Königin mitverantwortet, belegen die Umstände nach der Tötung Morolds. Gurmun, der König, verzichtet auf die Rache an Tristan hinsichtlich der Tötung seines vorvehtaere; er tut dies allerdings widerwillig, wie die folgenden Verse zeigen, und nur auf Betreiben der beiden Isolden, Königin und Tochter: ungerne sô verkiuse ich / iedoch verkiuse ich disen zorn, / sit die vouwen hânt verkorn (v. 10665-10667). Dabei rückt nicht zufällig die Sippenzughörigkeit Morolds zu Isolde in den Fokus, wenn er zu Isolde sagt: er gât dich mêre an danne mich. Môrolt dîn bruoder der was dir / nâher gesippe danne mir. hâstu'z umbe in varen lân, wiltû, sô hân ouch ich'z getân (v. 10649-10652). 
Implikationen gut vertraut. Die realen Eroberer, die Irland in Besitz nahmen, tendierten dazu, die Töchter alter regierender Familien zu heiraten, um so ihre Macht zu festigen (vgl. hierzu Edel 2001: 176). Das ist eben die Konstellation bei Gottfried: Der Königssohn Gurmun, in Afrika geboren (vgl. v. 5883), muss sich nach dem Tod seines Vater das Reich mit seinem Bruder teilen (vgl. v. 5886); mit römischer Legitimation überfällt und besiegt Gurmun Irland und wird dort gegen den Willen der Irländer - âne ir danc (v. 5918) - König. Er ist deshalb auf die Heirat mit einer reichen einheimischen Erbin angewiesen, das ist in diesem Fall die Schwester des irischen Herzogs Morold, der über Landbesitz und großen Reichtum (vgl. v. 5939) verfügt. Erst diese Heirat verhilft Gurmun zu Ansehen, erst jetzt wird er gefürchtet, d.h. anerkannt (vgl. v. 5931-5934). Dabei spielt auch eine Rolle, dass Isoldes Bruder Morold mit übermenschlichen Kräften ausgestattet ist, sein Name ist etymologisch mit dem Meer verbunden (vgl. Hammer 2007: 83 u. 88) und weist mit seiner vier manne craft (v. 6879) auf magischdämonische Aspekte der irischen Anderwelt (vgl. Hellgardt 2002: 169). Obwohl selbst an einer Herrscherposition interessiert (v. 5936f.), kommt es jetzt zu einer Allianz: Morold wird vorvehtaere (v. 5941) und ist »nun sozusagen für die (kriegerische) Außenpolitik Irlands zuständig« (Rinn 1996: 200); er treibt den Tribut ein, Geiseln nämlich aus Cornwall und England, dem Reich Markes. Diese Konstellation markiert ebenfalls politische Implikationen des frühen Irland. ${ }^{22}$

\subsection{Isolde und die irischen filid}

In der mediävistischen Forschung erregte vor allem Isoldes souveräne Rhetorik in der Gerichtsszene Erstaunen, die den betrügerischen Truchsess entlarvt und damit die Ehre des Reiches rettet. Gurmun hat daran keinen Anteil, er schiebt die Verantwortung der Königin zu: vrouwe, sprechet ir / vür iuch, vür Îsot und vür mich (v. 983of.). Classen brachte es auf den Punkt: »[T] he honor of the entire kingdom ist at stake, and it is the queen alone who can win the legal battle against the steward « (Classen 2004: 50), und weiter:

The assembly of Irish lords [...] does not exert any real influence and is actually ridiculed by the narrator [...]. The debate between the queen and the steward immediately demonstrates her absolute superiority and sovereignty over the steward because she does not even have to develop her own rhetorical strategy and can simply reiterate his own arguments [...] by satirically turning them into their opposite (ebd.: 49).

22 Der König herrschte über ein Volk (ir. túath), doch kam es "zu allen Zeiten zu politischer Machtkonzentration, zum Aufstieg einiger Könige über ihre unmittelbaren Nachbarn. Ein solcher mächtigerer König heißt in den Rechtstexten ruiri ('König von Königen`); er war nicht Herrscher über mehrere túatha, sondern hatte in einem persönlichen Abhängigkeitsverhältnis den rí túathe, der inm Tribut zahlte und Geiseln stellte“ (Richter 1996: 30). 
Isoldes satirische Rede meint die Replik auf den 30 Verse umfassenden misogynen Anwurf des Truchsess gegen alle Frauen, die das Böse für gut und das Gute für böse hielten (vgl. v. 9866-9896). Die Königin hält dagegen, dass seine Argumente so seien, als ob sie von Frauen erdacht wären, er habe vrouwen site (v. 9912) angenommen und der manne art (v. 9908) verloren. Am Ende ist der Truchsess Ziel von Spott und Hohn: sus nam der valsch ein ende / mit offenlîcher schende (v. 11364f.). Auch den Anstoß zur Aufdeckung der Lüge hatte diu wîse Isôt (v. 9404) gegeben, die ir tougenlîche liste (v. 9301) befragt und im Traum gesehen hatte, daz ez niht alsô geschah, / als der lantschal sagete (v. 9304f.).

Die ungewöhnliche Kombination von prophetischer Gabe (Wahrträume) sowie Schmähung und Herabsetzung des Gegners durch satirische Rede in aller Öffentlichkeit gehört nicht in das höfische Register, sondern markiert zweifellos einen fremden Raum, einen Raum, in dem die sogenannten filid (wörtlich >Seher<) beheimatet waren, Männer und eben auch Frauen, >learned women< des frühen Irland, die sich in der Prophetie sowie dem Verfassen von Lob- und Spottgedichten hervortaten. Die »stärkste Waffe des Dichters, dessen soziale Stellung es nicht erlaubte, mit physischer Gewalt zu kämpfen, lag im Bereich der Schmähungen«, es ging um die »Herabsetzung des Gegners« (Richter 1996: 27); filid waren Meinungsbildner, die die Machthaber beurteilten (vgl. Edel 2012: 133); im »Sinne einer Zensur auf die privilegierten Schichten « (Botheroyd/Botheroyd 1992: 120) konnten sie z.B. Wahrheit durchsetzen; sie waren zudem der Prophetie als Kunst des zukünftigen Wissens kundig (vgl. ebd.). Filid gehörten zur Gelehrtenklasse; das war eine »zahlenmäßig kleine, sozial aber hoch bedeutende Gruppe in der túath « (Richter 1996: 32), zu denen neben den Dichtern auch die Druiden, die Rechtsgelehrten und die Experten in Genealogie und Geschichte gehörten (vgl. ebd.). Filid nahmen innerhalb des frühen irischen Rechts eine Sonderstellung ein; ursprünglich hatten sie wie der König »den höchsten Ehrenpreis (enech, urspr. >Gesicht<, vergleichbar dem germ. Wergeld), waren also rechtlich nicht belangbar« (ebd.: 28). Eine verheiratete Frau hatte im Allgemeinen einen Ehrenpreis, der halb so groß war wie der ihres Gatten, weibliche filid wie auch andere >learned women< (z.B. Ärztin und Kunstschmiedin, die ökonomisch unabhängig waren) hatten einen eigenen Ehrenpreis (vgl. Edel 2001: 49). Das berühmteste Beispiel einer banfili, d.h. einer weiblichen fili, in der Literatur ist übrigens Fedelm, ${ }^{23}$ die banfili von Connacht, die Medb im >Rinderraub< die Zukunft bezüglich ihres Angriffs auf Ulster weissagt. ${ }^{24}$ Die Existenz histori-

23 | Zur unterschiedlichen Konturierung Fedelms in Rezension I und II siehe Enright 1996: $172 f$.

24 | Als Medb ihr begegnet, ist die Seherin gerade von Albion zurückgekommen (Albion ist der antike Name für die Britischen Inseln oder Großbritannien), wo sie filidecht studiert hat, das meint die traditionelle Ausbildung der filid, die eben auch eine Technik einschließt, die sie befähigt, in die Zukunft zu sehen (vgl. dazu Edel 2001: 39). Sie sitzt auf Mebds Wagendeichsel und webt mit einem Webschwert eine glänzende Borte aus finnruinne, d.h. blassgoldenen Bronzefäden. Die Beschreibung von Fedelm, ein schönes junges Mädchen, wiewohl bewaffnet wie ein Krieger, weist auf übernatürliche 
scher banfilid ist z.B. durch die Anzeige des Todes einer banfili im ersten Drittel des 10. Jahrhunderts in Annalen bezeugt (vgl. Clancy 1996: 46). ${ }^{25}$

Gottfrieds irische Königin gehört in diese Kategorie solcher >learned women<. Ihr Lehrer hat sie von Kind an unterrichtet in maneger guoten lêr, / mit manegem vremedem liste (v. 7712f.), in zahlreichen Wissenschaften also und vielen ungewöhnlichen Künsten. Diese vage Aussage hinsichtlich vremeder liste (die eigentlich die Zauberkunst, die Prophezeiung und die ungewöhnliche Redekunst meinen) ist bei Gottfried als politisch-pragmatisches Potential teilweise rationalisiert. Die Prinzessin, die gleich im Anschluss genannt wird, bedient dann nicht zufällig nur das textlogisch >eigene< Register. Denn die junge Isolde wird zwar von demselben Lehrer wie ihre Mutter unterrichtet, doch >nur< in buoch und seitspil (v. 7727) und erhält dann bekanntlich von Tristan auch noch eine gelehrt-künstlerische Ausbildung. Die Prinzessin ist eben keine banfili mehr, sie kann nicht weissagen (oder gar zaubern und heilen) - und das darf auch nicht anders sein: Die junge Isolde wird das textlogisch $>$ Fremde $<$, das zauberkundige Irland, verlassen, der höfische Markehof aber ist nicht anschließbar an vremede liste. Die junge Isolde ist in Cornwall dann Meisterin der >schönen< List als Bestandteil einer spezifisch höfischen Form der Konfliktlösung.

\subsection{Sonnen- und Mondmetaphorik als Superioritätssignal}

Die Frauen des irischen Königshofes werden im Zuge der Auffindung und Rettung Tristans nach seinem Drachenkampf in astronomischem Vokabular beschrieben: Königin, Prinzessin und Brangäne erscheinen als Morgenrot, Sonne und Vollmond. Auch in der zweiten Gerichtsszene spielt diese Sonnen- und Mondmetaphorik eine Rolle: Die drei Frauen sind wunderbar gekleidet, die Königin Isolde, daz vrôliche morgenrôt (v. 10866), führt ir sunnen an ir hant, / daz wunder von Îrlant (v. 10887f.), und Brangäne ist daz schoene volmaene (v.11082); die drei Frauen erscheinen »as the guiding stars or moon for Tristan and offer him salvation « (Classen 2004: 43). Das ist einerseits als Paradebeispiel adlighöfischer Statusrepräsentation zu lesen: »Licht oder gar Sonne [zählen] zu den häufigsten Metaphern der Ehre« (Röcke 1990: 46), und zudem dienen das Licht

Bezüge: Dass sie drei goldene Haarflechten hat, wovon zwei den Kopf umwinden, die dritte aber bis zur Wade herabhängt, sowie drei Pupillen in jedem Auge, "is a strong indication of her divine status" (Aldhouse-Green 1995: 149), und auch ihr gesprenkeltes Gewand impliziert anderweltliche Bezüge (vgl. ebd.). Auf Medbs Aufforderung, dass sie den Ausgang ihrer Unternehmung voraussagen solle, warnt sie dreifach (weil Medb sich weigert, der Prophezeiung zu glauben), dass sie Medb und ihre Verbündeten blutverschmiert sieht; nach der dritten Warnung singt sie eine zehnstrophige Prophezeiung in gebundener Sprache, in der sie Cú Chulainn (dem Medb unterliegen wird) und seine Helden bis in Einzelheiten hinein beschreibt.

25 | Die Dichterin starb entweder 934 oder 932 n.Chr., die Annalen nennen sie the female poet of Ireland، (banfile hÉrend; vgl. auch Edel 2001: 45). 
des Tages, primär die Sonne, sekundär aber auch das Licht der Nacht, der Mond, der Rechtswahrung und -sicherung (vgl. Wenzel 1988: 350f.).

Die Frauen-Dreizahl ist aus der Antike gut bekannt, sie war an den biblischen Bericht der drei Marien anschließbar, sie kann andererseits jedoch genauso den sanderen < Raum Gottfrieds assoziieren. Für Caples jedenfalls repräsentieren die beiden Isolden sowie Brangäne, als Dreiheit verstanden, die Celtic Triple Goddess (vgl. Caples 1975: 174); für Botheroyd gehört Isolde in die Tradition der großen keltischen Frauengestalten, denen letztlich die große Muttergöttin Modell stand (vgl. Botheroyd/Botheroyd 1992: 172). Wie dem auch sei: Es fällt immerhin auf, dass der irischen Göttin Brigit sehr spezifische Fähigkeiten zugeschrieben wurden, die auch Gottfrieds Königin kennt: Die Göttin galt als Seherin, Heilerin bzw. Ärztin und Patronin der Dichter (vgl. AldhouseGreen 1995: 198), sie wurde als banfili und Frau der Gelehrsamkeit verehrt (vgl. Weisweiler 1939: 251) und galt sowohl als einzelne wie auch als triple goddess. Der Name Brigit kommt vom keltischen Wort brig, das Macht und Autorität andeutet (vgl. Aldhouse-Green 1995: 196) und >Hoheit<, >Erhabenheit< meint (vgl. Botheroyd/Botheroyd 1992: 50); ihr unterstanden die aristokratischen Berufe der Druidenklasse, sie sorgte jedoch auch für Vieh und Ernte (vgl. ebd). Die Göttin Brigit kann sich in drei Gestalten entfalten, sie kann als dreifache Göttin in drei Verkörperungen erscheinen (vgl. ebd.) und erinnert dabei an die Matres oder Matronae (vgl. Lehmacher 1995: 237). Offenbar vermischten sich in ihrer Gestalt als ursprüngliche Muttergottheit auch Vorstellungen von Mond- und Sonnengöttin (vgl. ebd.), ja sie besaß »eine nicht zu unterschätzende Sonnenkomponente« (Botheroyd/Botheroyd 1992: 50), wobei überhaupt ein Zusammenhang von Muttergottheit und Sonne (vgl. Aldhouse-Green 1995: 114f.) zu beobachten ist (in einer interpretatio christiana wird die irische Göttin dann zur Heilligen umgebildet). Ein weiterer Aspekt: Brigit war die Tochter von Dagdha (>Guter Gott< oder >Guter Tagく), Anführer der Anderwelt, der die Jahreszeiten und Ernten regelt, ${ }^{26}$ König der Túatha Dé Dannann (vgl. Paton 1960: 11); diese Bezeichnung, die >Stämme der Göttin Danu< meint, ist ein jüngerer Name für die Bewohner der Anderwelt, die urspünglich síde (Ableitung der Wurzel >sed<, $>$ wohnen<) genannt wurden, ein Name, der schon um 700 belegt ist (vgl. Edel 2012: 151f.). Unter der Bezeichnung >Stämme der Göttin Danu< haben dann »die Klostergelehrten den alten Gottheiten einen Platz in ihrer chronologischen Geschichte Irlands eingeräumt« (ebd.: 152).

Nur Gottfried kennt die bifigurale Isoldekonzeption, in den Rettungsszenen (Auffindung und Heilung Tristans, Gerichtsszene) werden daraus drei Frauen. Die Polyvalenz der Frauen-Dreiheit konnte wie gesagt den >anderen $<$ Raum Gottfrieds markieren, denn die Kelten betrieben mit der Zahl drei »auffallend viel Aufwand; [...] alles, was mit dem Heiligen und Übernatürlichen in Verbindung stand [wurde] als Triade vermittelt« (Botheroyd/Botheroyd 1992: 89). So sind z.B. auch die drei Schwestern Ériu, Banba und Fódla die göttlichen Eponyme für Irland, die die Macht des Landes repräsentieren (vgl. MacKillop 1998: 29f., 169

26 | Vgl. zum vorgestellten Sachverhalt Edel 2012: 151. 
u. 211); sie besaßen zwar eigene Namen, alle aber verkörperten >Mutter Irland »Ihre drei Gatten [...] waren bedingt zu Eigenaktionen fähig, handelten aber im Grunde als ein König « (Botheroyd/Botheroyd 1992: 89). Als Triaden erscheinen übrigens auch die Kriegsdämoninnen Morrígan als >Alp-Königin ‘ im Sinne von $>$ Nachtmahr< (vgl. ebd.: 240), Bodb mit der Bedeutung >Krähe< und Nemain mit der Bedeutung >Panik<; alle drei konnten sich als Aaskrähen manifestieren (vgl. Edel 2012: 141). Diese Gestalten als eigene Kategorie der Anderwelt-Bewohner ${ }^{27}$ waren - anders als die göttliche Triade - nicht an den höfischen Code anschließbar und spielen deshalb auch im Tristan Gottfrieds keine Rolle.

\subsection{Der Drachenkampf: Übernahme des patriarchalen Systems}

Es ist nicht zu übersehen, dass kurz vor Abschluss der Irlandhandlung plötzlich die Männer das Sagen im textlogisch >fremden< Raum haben. Denn mit der Übergabe Isoldes an Tristan greifen zweifellos patriarchale Mechanismen: Der männliche Gewalthaber der Braut, ihr Vater König Gurmun, vollzieht jetzt das althergebrachte Eherecht der autoritativen Sippenvergabe, die Frauen werden nicht mehr gefragt und nicht gehört. Rabine (vgl. 2002) sieht in der Drachenepisode den entscheidenden Wendepunkt: Als Tristan den Drachen besiegt, muss ihm die Prinzessin übergeben werden. Dabei werden weder das höfische als dominierendes semiotisches System noch das Frauenregister als >Fremdes bedient, jetzt greift ein drittes Register, das mythische: Der Drachenbezwinger bekommt die Königstochter. Dies gehört eigentlich in die Heldenepik mit ihrer heroischen Maskulinität und steht im größtmöglichen Abstand zu Gottfrieds $>$ Fremdem<; ein zweiter fremder Raum dominiert also zu diesem Zeitpunkt die Handlung. Die Frage, warum überhaupt ein drittes Register eröffnet wird, kann mit der sujethaften Kollision ${ }^{28}>$ Tristan und Drache< der Stofftradition beantwortet werden.

In der >Badszene < zuvor war ebenso eine Konkurrenz ausagiert worden, hier mit dem Sieg des Höfischen, des textlogisch >Eigenen< also. Denn als Tristan im Bad sitzt und die Prinzessin anhand der schadhaften Schwertscheide Tristan als Mörder ihres mütterlichen Onkels Morold erkennt, will sie bekanntlich den wehrlosen Tristan mit dessen eigenem Schwert durchbohren (vgl. v. 10180), doch die Königin erinnert an ihr Schutzversprechen (vgl. v. 10213f.). Sofort wird beteu-

27 | Ihre unheilvolle Wirkung war psychologischer Natur: Morrigán etwa hetzt in der Nacht die Armeen gegeneinander auf (vgl. Dillon 1972: 11), auch war die Erscheinung der drei Dämoninnen so, dass sie den tapfersten Krieger entmutigen konnten, und ihr Heulen derart, dass sie das Blut gefrieren ließen und den Krieger dazu bringen konnten, dass er die Waffen wegschleuderte und das Schlachtfeld verließ (vgl. AldhouseGreen 1995: 42). Ein Beispiel aus dem 'Rinderraub،: Nemain brought confusion on the host and they fell trembling in their arms under the points of their spears and weapons, so that an hundret warriors of them fell dead in the midst of their camp and quarters at the fearfulness of the shout they raised on high ([Táin Bó Cuailnge] 1914: 308). 28 | Vgl. zu sujethaften (und sujetlosen) Texten Lotmann 1993: 336-341. 
ert, dass die junge Isolde aufgrund ihrer süezen wîpheit Tristan ohnehin nicht hätte erschlagen können, obgleich zorn unde wîpheit (v.10260) in ihr kämpften. Wenn die wîpheit über den zorn siegt, dann siegt die »aufgeklärte Norm, die in der Erziehung grundgelegte Verbindung von Vernunft (sin) und Etikette (schoene site) [...] über die archaischen Obsessionen und Gewaltmechanismen « (Küsters 1986: 163). Nach Rabine bedeutet Isoldes (höfischer) Racheverzicht jedoch eine Fehlleistung, sie wäre zur Blutrache verpflichtet gewesen; in dem Moment, als sie Tristan verschont, verrate sie »her sacred tie to her uncle Morold« (Rabine 2002: 6of.):

By bringing into opposition 'womanhood' and ranger', this passage gives both terms a new meaning in relation to each other. Womanhood now excludes anger, and no longer connotes the women of Irish legend, but a more subdued type of feminity, whil anger, formerly associated with the ancient bisexual clanic duty of vengeance, is now reserved for 'manhood، (ebd.: 65).

Ob man dem nun folgen kann oder nicht, ${ }^{29}$ fest steht, dass »when the sister's son or the maternal uncle was killed, the duty of vengeance devolved upon the other, according to the Ancient Laws of Ireland « (Farnsworth 1966: 231). In der $>$ Badszene < dominiert also das >Eigene < das textlogisch >Fremde< erster Ordnung, in der Brautübergabe setzt sich dann ein Fremdes zweiter Ordnung durch, das über seine unverkennbar patriarchale Struktur jedoch an das >Eigene anschließbar ist. Die mythische Drachentötung dominiert am Ende Irlands >Frauenpower<.

\section{EXKLUSION}

In Eilharts Version ist das textlogisch >Fremde< (erster Ordnung) des Gottfried'schen Texts vollständig ausgeblendet, es kommt diesbezüglich zu keiner interkulturellen Begegnung. Die irische Königin ist hier eben nicht >political leader and diplomat<, sie ist nicht die banfili-ähnliche wortmächtige Prophetin (Tristan nimmt die Verteidigung bei Gericht in Sachen Truchsess selbst in die Hand), sie ist auch nicht die zauberkundige Heilerin, die Tristan zwei Mal das Leben rettet, sie wird nur knapp erwähnt bei der Bereitung des Minnetranks. Ebenso

29 | Für den Keltologen Stefan Zimmer, den ich danach befragte, stellt sich die Sache etwas anders dar. Er bezieht sich dabei auf Kelly (vgl. Kelly 1991: 127), der hinsichtlich der Blutrache im frühen Irland darlegte, dass zunächst eine Verpflichtung besteht, die Schuld aus Totschlag/Mord mittels der üblichen Verhandlungen und Zahlungen beizulegen. Erst wenn der Schuldige bzw. seine Familie etc. sich weigern zu zahlen, sind die Verwandten verpflichtet, Blutrache zu üben: "If a non-paying killer is at large, the vitcim's kinsmen are obliged to carry out a blood-feud to exact vendeance (dígal) on behalf oft he dead man" (ebd.). 
existiert bei Eilhart kein glänzendes Dreigestirn der irischen Frauen mit deren bemerkenswerter Sonnen- bzw. Mondmetaphorik. Warum ist das so?

Eilharts erzählte Welt ist zweifellos die einer laikalen Kriegergesellschaft; diese ist im Gaunt'schen (vgl. Gaunt 1995: 23) Sinne als Träger monologischer Maskulinität im homosozialen Raum vermittelt; weibliche Stimmen fehlen bzw. werden nicht gehört. Während also bei Gottfried das textlogisch >Fremde < in den Text integriert ist, freilich unter der Prämisse bestimmter Ausschlussregeln: Das $>$ Fremde< ist räumlich ausgelagert (Insel), der Held, nicht der König, ist der Unterhändler, das >Fremde < ist offen für Prozesse der kulturellen Infiltration durch das >Eigene<, auch bildet die Superiorität der >fremden < Frauen keinen völligen Kontrast zur Idealgestalt einer höfischen vrouwe - ist das bei Eilhart undenkbar: Das >Fremde< der Gottfried'schen Dramaturgie ist in Eilharts Version in keinem Fall an deren >Eigenes< (monologische Männlichkeit innerhalb einer laikalen Kriegergesellschaft) anschließbar, eben auch dann nicht, wenn es als Heterotopie inszeniert wäre. ${ }^{30}$

\section{LiTERATUR}

Aldhouse-Green, Miranda (1995): Celtic Goddesses. Warriors, Virgins and Mothers. London.

Bitel, Lisa (1996): Land of Women. Tales of Sex and Gender from Early Ireland. New York. Botheroyd, Paul F./Brotheroyd, Sylvia (Hg.; 1992): Lexikon der keltischen Mythologie. München.

Bowen, Charles (1974): Great-Bladdered Medb. Mythology and Invention in the Táin Bó Cúailnge. In: Eire-Ireland 10, H. 4, S. 14-34.

Caples, Cynthia Barret (1975): Brangaene and Isold in Gottfried von Strassburg's ,Tristanı. In: Colloquia Germanica 9, S. 39-50.

Clancy, Thomas Owen (1996): Women Poets in Early Medieval Ireland. Stating the Case.

In: Christine E. Meek/ Katherine Simms (Hg.): „Fragility of her Sex?" Medieval IrishWomen in their European Context. Dublin, S. 43-72.

Clarus, Ingeborg (1991): Keltische Mythen. Der Mensch und seine Anderswelt. Olten.

Classen, Albrecht (2004): Female Agency and Power in Gottfried von Straßburg's

Tristan،. The Irish Queen Isolde: New Perspectives. In: Tristania 23, S. 39-60.

Dillon, Myles ( $\left.{ }^{5} 1972\right)$ : Early Irish Literature. Chicago.

Edel, Doris (2001): The Celtic West and Europe. Studies in Celtic Literature and the Irish Church. Cornwall.

Dies. ( $\left.{ }^{3} 2012\right)$ : Keltische Literatur. In: Stefan Zimmer (Hg.): Die Kelten. Mythos und Wirklichkeit. Stuttgart, S. 122-160.

30 | Für ausgesprochen engagierte Hilfe bei der Erstellung der Publikation danke ich meinen Mitarbeitern, allen voran Herrn Tobias Klich, der mich mit wertvollen Literaturhinweisen versorgte und sich als kompetenter Diskutant erwies; zu danken ist jedoch ebenso Alexander Kastenmayer sowie Isabell Hesse; Herrn Johannes Hütten danke ich für seine wie immer kompetente redaktionelle Korrekturarbeit. 
Egeler, Matthias (2012): Some Thoughts on 'Goddess Medb، and her Typological Context. In: Zeitschrift für celtische Philologie 59, S. 67-96.

Enright, Michael J. (1996): Lady with a Mead Cup. Ritual, Prophecy and Lordship in the European Warband from Latène to the Viking Age. Dublin.

Farnsworth, William 0. (1966): Uncle and Nephew in the Old French Chansons de Geste. A Study in the Survival of Matriarchy. New York.

Foucault, Michel (1992): Andere Räume. In: Karlheinz Barck u.a. (Hg.): Aisthesis. Wahrnehmung heute oder Perspektiven einer anderen Ästhetik. Leipzig, S. 34-46.

Gaunt, Simon (1995): Gender and Genre in Medieval French Literature. Cambridge.

Geertz, Clifford (1973): The Interpretation of Cultures. Selected Essays. New York.

Ders. (1987): Dichte Beschreibung. Beiträge zum Verstehen kultureller Systeme. Übers. v. Brigitte Luchesi u. Rolf Bindemann. Frankfurt a.M.

Gottfried von Straßburg (2011): Tristan. 2 Bde. Hg. v. Walter Haug u. Manfred Günter Scholz. Berlin.

Greene, David (1959): Táin Bó Cúailnge. In: Myles Dillon (Hg.): Irish Sagas. Dublin, S. 94-106.

Gutjahr, Ortrud (2010): Interkulturalität als Forschungsparadigma der Literaturwissenschaft. Von den Theoriedebatten zur Analyse kultureller Tiefensemantiken. In: Dieter Heimböckel/Irmgard Honnef-Becker/Georg Mein/Heinz Sieburg (Hg.): Zwischen Provokation und Usurpation. Interkulturalität als (un)vollendetes Projekt der Literatur- und Sprachwissenschaften. München, S. 17-39.

Hammer, Andreas (2007): Tradierung und Transformation. Mythische Erzählelemente im ,Tristan، Gottfrieds von Strassburg und im ,Iwein، Hartmanns von Aue. Stuttgart.

Heimböckel, Dieter/Mein, Georg (2010): Zwischen Provokation und Usurpation oder Nichtwissen als Zumutung des Fremden. Zur Einleitung des Bandes. In: Dieter Heimböckel/Irmgard Honnef-Becker/Georg Mein/Heinz Sieburg (Hg.): Zwischen Provokation und Usurpation. Interkulturalität als (un)vollendetes Projekt der Literatur- und Sprachwissenschaften. München, S. 9-14.

Hellgardt, Ernst (2002): Tristanroman und 'Vọlsunga Saga،. Mythos, Magie und Liebe. Zwei mittelalterliche Paradigmen zum Thema ,Liebe als Passion. In: Christoph Huber/Victor Millet (Hg.): Der Tristan, Gottfriends von Straßburg. Symposion Santiago de Compostela, 5.-8. April 2000. Tübingen, S. 167-198.

Jackson, Kenneth Hurlstone (1964): The Oldest Irish Tradition. A Window on the Iron Age. Cambridge.

Kellermann-Haaf, Petra (1986): Frau und Politik im Mittelalter. Untersuchungen zur politischen Rolle der Frau in den höfischen Romanen des 12., 13. und 14. Jahrhunderts. Göppingen.

Kelly, Fergus ( $\left.{ }^{2} 1991\right)$ : A Guide to early Irish law. Dublin.

Küsters, Urban (1986): Liebe zum Hof. Vorstellungen und Erscheinungsformen einer "höfischen" Lebensordnung in Gottfrieds ,Tristanı. In: Gert Kaiser/Jan-Dirk Müller (Hg.): Höfische Literatur, Hofgesellschaft, höfische Lebensformen um 1200. Kolloquium am Zentrum für Interdisziplinäre Forschung der Universität Bielefeld (3. bis 5. November 1983). Düsseldorf, S. 141-176. 
Lehmacher, Gustav (1995): Die Götting Brigit. In: Kurt Derungs (Hg.): Keltische Frauen und Göttinnen. Matriarchale Spuren bei Kelten, Pikten und Schotten. Grenchen b. Solothurn, S. 236-244.

Lotman, Jurij Michailowitsch (1990): Über die Semiosphäre. In: Zeitschrift für Semiotik 12, S. 287-305.

Ders. ( $\left.{ }^{4} 1993\right)$ : Die Struktur literarischer Texte. Übers. v. Rolf-Dietrich Keil. München. MacKillop, James (Hg.; 1998): Dictionary of Celtic Mythology. Oxford/ New York. Mälzer, Marion (1991): Die Isolde-Gestalten in den mittelalterlichen Tristan-Dichtungen. Ein Beitrag zum diachronischen Wandel. Heidelberg.

Ó hUiginn, Ruairí (1993): Zu den politischen und literarischen Hintergründen der Táin Bó Cuailnge. In: Hildegard L.C. Tristram (Hg.): Studien zur Táin Bó Cuailnge. Tübingen, S. 133-157.

Paton, Lucy Allen ( $\left.{ }^{2} 1960\right)$ : Studies in the fairy mythology of Arthurian romance. New York.

Patterson, Nerys Thomas (1991): Cattle-Lords and Clansmen. Kinship and Rank in Early Ireland. New York/ London.

Rabine, W. Leslie (2002): Love and the New Patriarchy. Tristan and Isolde. In: Joan Tasker Grimbert (Hg.): Tristan and Isolde. A Casebook. New York/London, S. 37-74. Rasmussen, Ann Marie (2003): The Female Figures in Gottfried's Tristan and Isolde. In: Will Hasty (Hg.): A Companion to Gottfried von Strassburg's "Tristan«. Rochester, S. 137-157.

Richter, Michael (1996): Irland im Mittelalter. Kultur und Geschichte. München.

Rinn, Karin (1996): Liebhaberin, Königin, Zauberfrau. Studien zur Subjektstellung der Frau in der deutschen Literatur um 1200. Göppingen.

Röcke, Werner (1990): Im Schatten des höfischen Lichtes. Zur Trennung von Öffentlichkeit und Privatheit im mittelalterlichen Tristan-Roman. In: Walter Gebhard (Hg.): Licht. Religiöse und literarische Gebrauchsformen. Frankfurt a.M., S. 37-75.

Ross, Anne (1967): Pagan Celtic Britain. London.

Semmler, Hartmut (1991): Listmotive in der mittelhochdeutschen Epik. Zum Wandel ethischer Normen im Spiegel der Literatur. Bielefeld.

Sterling-Hellenbrand, Alexandra (2001): Topographies of Gender in Middle High German Arthurian Romance. New York.

[Táin Bó Cuailnge] (1914): The Ancient Irish epic Tale Táin Bó Cúalnge. The Cualnge Cattle-Raid. Übers. v. Joseph Dunn. London.

Thurneysen, Rudolf (1935): Das keltische Recht. In: Ders.: Gesammelte Schriften, Bd. III. Hg. v. Patrizia de Bernardo Stempel u. Rolf Ködderitzsch. Tübingen, S. 263286.

Ders. (1936): Cāin lānama, "Die Regelung der Paare». In: Ders.: Gesammelte Schriften, Bd. III. Hg. v. Patrizia de Bernardo Stempel u. Rolf Ködderitzsch. Tübingen, S. 287365.

Van Gennep, Arnold ( ${ }^{32005): ~ U ̈ b e r g a n g s r i t e n . ~ A u s ~ d e m ~ F r a n z . ~ v . ~ K l a u s ~ S c h o m b u r g ~ u . ~}$ Sylvia M. Schomburg-Scherff. Frankfurt a.M.

Weisweiler, Josef (1939): Die Stellung der Frau bei den Kelten und das Problem des "keltischen Mutterrechts". Aus: Zeitschrift für celtische Philologie 21, H. 2, S. $205-$ 279. 
Wesel, Uwe (1980): Der Mythos vom Matriarchat. Über Bachofens Mutterrecht und die Stellung von Frauen in frühen Gesellschaften vor der Entstehung staatlicher Herrschaft. Frankfurt a.M.

Wenzel, Horst (1988): Öffentlichkeit und Heimlichkeit in Gottfrieds ITristan`. In: Zeitschrift für deutsche Philologie 107, S. 335-361. 
